
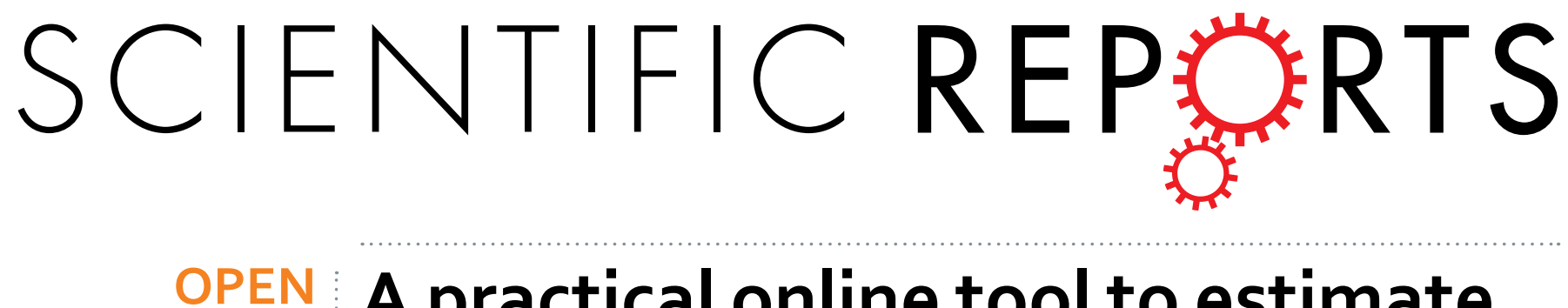

\title{
A practical online tool to estimate antiretroviral coverage for HIV infected and susceptible
}

Received: 29 March 2016

Accepted: 06 June 2016

Published: 24 June 2016

\section{populations needed to reduce local HIV epidemics}

\author{
Antoine Chaillon ${ }^{1}$, Martin Hoenig| ${ }^{1,2,3}$, Sanjay R. Mehta ${ }^{1,4}$, Nadir Weibel ${ }^{5}$, Susan J. Little ${ }^{1}$ \& \\ Davey M. Smith ${ }^{1,4}$
}

It remains unclear what proportions of HIV-infected and uninfected people should receive effective antiretroviral therapy (ART) to control local HIV epidemics. We developed a flexible model to evaluate the impact of treatment as prevention (TasP) and pre-exposure prophylaxis (PrEP) on HIV incidence in local communities. We evaluated this tool for determining what TasP and PrEP targets are needed to substantially reduce the HIV epidemic in San Diego, which is predominately comprised of men who have sex with men. By increasing the proportion of HIV-infected individuals on ART from $30 \%$ to $50 \%$, 686 new infections would be prevented over five years in San Diego. By providing PrEP to 30\% of MSM to the age group that account for $90 \%$ of local HIV incident cases (21-52 years), we could prevent 433 infections over five years. When combining these initiatives, a PrEP coverage rate of $40 \%$ and TasP coverage rate of $34 \%$ would be expected to decrease the number of new infections by over half in one year. This online tool is designed to help local public health planners and policy makers to estimate program outcomes and costs that may lead to better control of their local HIV epidemics.

It is becoming increasingly clear that we may now have the tools to end the HIV epidemic, especially with broader use of antiretroviral therapy (ART) ${ }^{1}$. Beyond improving the lifespan and well-being of HIV-infected individuals, ART can also be used as (1) treatment as prevention $(\text { TasP })^{2},(2)$ pre-exposure prophylaxis $(\operatorname{PrEP})^{3},(3)$ post-exposure prophylaxis and (4) microbicide. The effectiveness of ART to reduce HIV incidence in a population, however, is limited by its uptake among those who are infected (i.e. TasP) and those who are susceptible (i.e. PrEP, PEP, and microbicides). This is especially poignant since only about $25 \%$ of HIV-infected people in the United States ${ }^{4,5}$ and $30 \%$ in San Diego ${ }^{6}$ now are diagnosed and on suppressive ART, and the number of highly susceptible HIV-uninfected people on PrEP is considerably lower ${ }^{7}$. The proportions of HIV-infected individuals on effective ART and susceptible HIV-uninfected individuals on PrEP that are needed to control an epidemic in a population remain unknown.

Empirical and deterministic models have been developed by several groups to evaluate the impact of voluntary HIV testing and immediate $\mathrm{ART}^{8}$, the impact on HIV prevalence and incidence of interventions that decrease disparities ${ }^{9}$ or cost-effectiveness of treatment and prevention ${ }^{10,11}$. While these studies are highly relevant to determine the theoretical impact of initiatives, they are complex and less conducive for adjustments for local HIV epidemics, especially when quickly comparing the adjustment of epidemic or initiative parameters to suit local efforts. In this setting, simple mathematical models using empirical parameters, as provided here, may help provide targets of TasP and PrEP coverage rates to reduce HIV incidence in local populations.

\footnotetext{
${ }^{1}$ Division of Infectious Diseases, University of California, San Diego, La Jolla, California, United States of America. ${ }^{2}$ Section of Infectious Diseases and Tropical Medicine, Department of Internal Medicine, Medical University of Graz, Graz, Austria. ${ }^{3}$ Division of Pulmonology, Department of Internal Medicine, Medical University of Graz, Graz, Austria. ${ }^{4}$ Veterans Affairs San Diego Healthcare System, San Diego, California, United States of America. ${ }^{5}$ Department of Computer Science and Engineering, University of California San Diego, California, United States. Correspondence and requests for materials should be addressed to A.C. (email: achaillon@ucsd.edu)
} 
Focusing on a community-based response, we developed an intuitive and flexible tool to estimate the potential impact of TasP and PrEP coverage rates on HIV incidence in a population. We evaluated our tool on the HIV epidemic in San Diego, using previously measured local HIV epidemic parameters ${ }^{12}$; however, the tool can be adapted to any population that has accurate estimates of epidemic parameters. We believe that such a tool may help to provide estimates of what proportion of HIV-infected and uninfected people in a local population need to be covered with ART (TasP and PrEP) to most efficiently control an epidemic.

\section{Methods}

This study aimed to create a practical tool to estimate the potential impact and costs of combined PrEP and TasP strategies on HIV incidence in a local population. We then evaluated this tool in the setting of the HIV epidemic in San Diego. To provide maximal flexibility and utility, we limited the number of input variables and proposed default parameters based on published data. Since local conditions can influence each of these variables, we designed our tool (http://gtzero.ucsd.edu/) to allow parameters to be adjusted.

Model Description. Number of new HIV infections. We used a standard crude rate of growth model to derive the number of new HIV infections over time in a given population. The population of viremic HIV-infected individuals was defined from the initial population size $\left(\mathrm{P}_{0}\right)$ based on a TasP coverage rate $(f)$ with an average TasP efficacy of $80 \%{ }^{13}$ for a HIV prevalence $(\pi)$. Hence:

$$
P(\text { viremic })=\pi \times(1-0.8 \times f) \times P(o)
$$

The population of susceptible individuals $P$ (susceptible) was estimated based on initial population size $P(o)$ and the HIV prevalence $(\pi)$. Assuming a PrEP coverage rate $(\partial)$ with an average PrEP efficacy of $70 \%^{3}$, $P($ susceptible) would be:

$$
P(\text { susceptible })=(1-0.7 \times \partial) \times[P(o)-\pi \times P(o)]
$$

Considering results of previous studies to estimate the HIV transmission risk per sex act (ß) for condomless receptive anal intercourse (CRAI) and condomless insertive anal intercourse (CIAI) ${ }^{14}$, we first estimated the overall transmission risk per condomless sexual act as:

$$
\left.ß=\beta_{\text {CRAI }}{ }^{*} \text { (proportion of RAI }\right)+\left(\beta_{\text {CIAI }} \text { *proportion of IAI }\right) \times(1-\mu)
$$

where $\mu=$ the frequency of condom use.

Here, the proportion of insertive and receptive sex acts indicate the probability that, for a given sex act between an HIV-positive partner and an HIV-negative partner, the HIV-negative partner is the receptive partner (or the insertive partner).

Then calculated the probability of transmission over $(\mathrm{C})$ acts using the standard binomial formula to estimate the cumulative risk over c exposures $B(c)^{15}$ :

$$
ß(c)=1-(1-\beta)^{c}
$$

Crude Cost Estimates. Next, we incorporated costs into our calculations in relation to infections averted. Since prices change, the provided tool allows TasP and PrEP costs to be adjusted. Assuming that cost estimates for TasP was $\$ 24,000$ (in 2016 US dollars) from ${ }^{16}$ and for PrEP was $\$ 10,300 /$ year from ${ }^{17}$, which included costs of recommended drugs (combination oral emtricitabine/tenofovir disoproxil fumarate) and clinical monitoring, considering U.S. inflation from 2010 to 2016 and fluctuation of costs over time due to multiple other factors among which manufacturer costs, third party payor negotiations, generic pricing, etc we derived the annual gross costs of TasP and PrEP as:

$$
\begin{gathered}
\operatorname{Cost}(\operatorname{Tas} P)=24,000 \times \pi \times P(t) \times f \\
\operatorname{Cost}(\operatorname{PrEP})=10,300 \times \pi \times P(o) \times(1-\pi) \times \partial
\end{gathered}
$$

Hence, the overall gross cost of combined TasP and PrEP initiative can be estimated as the sum of (1) and (2). We also took into account the annual costs saved by new infection averted (NIA) as:

$$
\operatorname{Cost}(N I A)=24,000 \times N I A \times f
$$

Derived from (1) to (3), the annual net cost for a given TasP coverage rate $(f)$ and PrEP coverage rate $(\partial)$ was defined as:

$$
\operatorname{Cost}(\operatorname{Tas} P+\operatorname{PrEP})=\operatorname{Cost}(\operatorname{Tas} P)+\operatorname{Cost}(\operatorname{PrEP})-\operatorname{Cost}(N I A)
$$

San Diego HIV Epidemic. We evaluated our tool for the HIV epidemic in San Diego using latest available data (Table 1$)^{18}$. Since the San Diego epidemic is largely centered among MSM ( $\left.>80 \%\right)$, we focused our model on the MSM epidemic in San Diego, considering a total of 56,000 MSM individuals $\left(P_{0}\right)$ and an HIV prevalence $(\pi)$ of $20 \%{ }^{19}$. 


\begin{tabular}{|c|c|c|c|}
\hline Variable & Definition & $\begin{array}{l}\text { Estimates for } \\
\text { MSM in San } \\
\text { Diego }\end{array}$ & Ref. \\
\hline \multicolumn{4}{|c|}{ HIV epidemic Estimates } \\
\hline$\beta$ & $\begin{array}{l}\text { Risk of HIV transmission per sexual act } \\
=\left[\left(\beta_{\mathrm{RAI}} * \text { proportion of } \mathrm{RAI}\right)+\left(B_{\mathrm{IAI}} * \text { proportion of IAI }\right)\right] *(1-\mu) \\
\text { where: } \\
-B(\mathrm{CRAI})=\text { condomless receptive anal intercourse } \\
-B(\mathrm{CIAI})=\text { condomless insertive anal intercourse } \\
-\mu=\text { Frequency of condom use } \\
-C=\text { number of sexual exposure per year }\end{array}$ & $\begin{array}{c}0.0138 \\
0.0011 \\
60 \%^{*} \\
10,20 \text { and } 30^{*}\end{array}$ & $\begin{array}{l}14 \\
14\end{array}$ \\
\hline$P_{0}$ & Initial total population size of MSM & 56,000 & \\
\hline$\pi$ & HIV prevalence & $20 \% *$ & 19 \\
\hline$P_{(\text {susceptible) }}$ & Initial population size of HIV exposed individuals & 52,920 & \\
\hline$\partial$ & PrEP coverage rate & Negligible* & \\
\hline$f$ & TasP coverage rate & $30 \% *$ & \\
\hline Efficacy of TasP & Percentage of individuals on ART who achieved viral suppression (TasP) & $80 \%$ & 13 \\
\hline Efficacy of PrEP & Percentage of individuals on PrEP who were considered protected & $70 \%$ & 3 \\
\hline \multicolumn{4}{|l|}{ Cost Estimates } \\
\hline TasP & Average annual cost of HIV care & $24,000 \mathrm{USD} /$ year & 16 \\
\hline PrEP & Average annual cost of PrEP (FTC/TDF)\# & $10,300 \mathrm{USD} /$ year & 17 \\
\hline
\end{tabular}

Table 1. Variables used to evaluate the San Diego HIV epidemic among men who have sex with men (MSM). ART: Antiretroviral Therapy; PrEP: Pre Exposure Prophylaxis; TasP: Treatment as Prevention; FTC/ TDF: combination oral emtricitabine/tenofovir disoproxil fumarate. *Adjustable variables; ${ }^{*}$ Annual cost of PrEP based on CDC recommendations for care, including drug costs, physician visits and laboratory testing ${ }^{17}$.

Coverage rate of effective ART among HIV-infected individuals. Currently, around $30 \%$ of persons living with HIV infection in the United States are prescribed ART and achieve viral suppression ${ }^{13}$, which is similar to data for San Diego ${ }^{18}$.

Efficacy of TasP. Use of ART can reduce HIV transmission by $92-98 \%{ }^{2,20}$. Other studies have less optimistic estimates $^{21}$, so we set the efficacy of TasP at $80 \%$, but have also included a sensitivity analysis for this parameter.

Efficacy of PrEP. Based on the iPrEX study ${ }^{3}$, the efficacy of PrEP in men who, by self-report and pill count, took the drugs more than $90 \%$ of the time was $73 \%$, so we conservatively estimated an average of $70 \%$ efficacy of PrEP to prevent HIV infection in among MSM in San Diego. Of note, the overall efficacy of belonging to the active PrEP arm in the iPrEX study was $44 \%$ and local conditions are likely to vary; therefore, the model allows this parameter to be modified directly by the user.

HIV transmission risk per sex act ( $\beta$ ). The risk of HIV per sexual act varies among risk groups. For San Diego, we used estimates from a recent review where MSM risk was estimated at 138 infections per 10,000 exposures for CRAI and 11 per 10,000 exposures for $\mathrm{CIAI}^{14}$. For the following population-based analyses, we considered an equal proportion of insertive and receptive acts (1:1).

Number of sex acts with casual partners per year $(C)$. Since the San Diego HIV epidemic is predominately a sexual risk epidemic among MSM and we did not have an empiric measurement of the average number of sexual contacts with casual partners $(C)$ among MSM in San Diego, we stratified our analyses by the mean number of sex acts with a casual partner (from 10 to 30 per year), which encompasses previous estimates provided by an Australian study that followed 1,427 MSM over 6 years ${ }^{22}$.

Condom use. Since condom use reduces sexual risk, we included estimates of condom use based on data from San Diego MSM reported in 4 categories: i) 'never' (0\%), ii) 'sometimes' (1-50\%), iii) 'mostly' (51-99\%) and iv) 'always' $(100 \%)^{12}$ (Fig. S1). From these data, we estimated an average condom use of $60 \%$ for anal sex.

Age targeted PrEP. Targeting PrEP to those at highest risk would likely be more efficient ${ }^{23}$. To evaluate if PrEP can be targeted to reduce HIV incidence while reducing costs, we estimated: (i) age distribution of men in San Diego from ${ }^{24}$, (ii) number of MSM in San Diego $\left(P_{0}\right)$ from, and ${ }^{18}$ (iii) young key MSM population in San Diego as the age group who account for $90 \%$ of new HIV infections from ${ }^{12}$ Hence, MSM in San Diego aged between 21 and 52 years old accounted for $46.3 \%$ of all $\mathrm{MSM}^{24}$ but represented $90 \%$ of all new HIV infection (Fig. S2).

Since $10 \%$ of at risk MSM will not have received any $\operatorname{PrEP}(\partial=0)$ as they do not fulfill criteria established for the $90 \%$ at highest risk, we defined the number of new HIV infections with PrEP targeted by age as:

$$
N(\text { targeted PrEP })=0.9 * N(t)+0.1 * N_{2}(t)
$$


where $N_{1}(t)$ is the number of new HIV infections for a given PrEP coverage rate $(\partial)$ and $N_{2}(t)$ is the number of new HIV infections without $\operatorname{PrEP}(\partial=0)$.

We also defined the cost estimates for PrEP targeted based on age as:

$$
\begin{aligned}
\text { Cost }(\text { targeted PrEP })= & \operatorname{Cost}(\operatorname{PrEP}) \times\left(\frac{\text { Population at High Risk }}{\text { Total Population }}\right) \\
& \times \text { No. of susceptible individuals }
\end{aligned}
$$

Hence in San Diego:

$$
\text { Cost }(\text { targeted } \operatorname{PrEP})=\operatorname{Cost}(\operatorname{PrEP}) \times 0.463 \times \text { No. of susceptible individuals }
$$

Sensitivity Analyses. To evaluate the impact on HIV incidence and costs of combined TasP and PrEP with and without PrEP targeted on age, we performed sensitivity analysis on: i) non-targeted PrEP coverage versus TasP coverage rates, and ii) age targeted PrEP coverage versus TasP coverage rates. We described the number of new HIV infection after one and five years and the cost per NIA for combined PrEP and TasP prevention strategies (i.e. $\frac{\text { Total cumulative Cost of PrEP and TasP }}{\text { Number of new HIV infection averted }}$ ). We also evaluated the potential impact of sexual risk compensation on the number of new HIV infection via sensitivity analysis based on PrEP coverage rate $(\partial)$, condom use frequency, and the average number of sexual acts with casual partner per year $(C)$.

Role of the funding source. The funding source had no role in the design and conduct of the study; collection, management, analysis, and interpretation of the data.

\section{Results}

It has been touted that local HIV epidemics can be controlled with TasP and $\mathrm{PrEP})^{3}$, but it is not clear what rates of TasP and PrEP are needed. To this end, we created and evaluated a tool that can provide estimates of TasP and PrEP coverage rates needed to reduce HIV incidence. We present this tool utilizing parameters for the HIV epidemic in San Diego, California, but the flexibility of the tool should allow for any local epidemic driven primarily by sexual risk to be evaluated.

HIV in San Diego. Around 10,710 HIV-infected individuals live in San Diego, California ${ }^{18}$. The vast majority of these individuals are MSM $(82 \%)^{18}$, and around $30 \%$ are receiving ART with suppressed viral loads ${ }^{13}$, while the proportion of susceptible MSM receiving PrEP is likely negligible ${ }^{25}$. Based on a mean of 20 sex acts per year, the cumulative number of newly infected individuals in San Diego would be expected to grow by 449 in one year and 2,291 in five years (Table S1). These numbers are congruent with local estimates reported by the Health and Human Services Agency of San Diego County in ref. 18.

Impact of TasP. In San Diego, if TasP coverage rate increased from the current rate of $30 \%$ to $40 \%$ coverage (Table S1), then 344 new infections could be averted over 5 years, and at 50\% TasP coverage rate 686 new infections could be averted (Fig. 1A, see*, Table S1). Gross cost estimates of increasing TasP from 30\% to 50\% coverage would be $\$ 286$ million over 5 years (Fig. S3A, Table S2). Interestingly, if TasP coverage rate was $50 \%$, then gross annual costs of TasP would be estimated to decrease after 10 years (Fig. S4).

Impact of PrEP. Considering a scenario of 30\% TasP coverage rate and an average of 20 sex acts per year, increasing PrEP coverage rate to 20\% of all MSM in San Diego would prevent 321 new HIV infections after 5 years compared to a scenario without PrEP (Fig. 1B, see*, Table S1). Increasing PrEP coverage rate from $20 \%$ to $50 \%$ all MSM in San Diego would avert 481 more infections (i.e. 1810 NIA compared to no PrEP, Fig. 1B, see*, Table S1). The cost of covering 30\% of all MSM in San Diego would cost $\$ 584$ million over 5 years (Fig. S5, see*, Table S2). Unlike for TasP, the costs of PrEP did not decrease with higher rates of PrEP coverage rate or over time.

PrEP Targeted by Age. While MSM are the main risk group in San Diego, the risk of infection is not uniform $^{26}$. We estimated that $90 \%$ of all new HIV infection among MSM in San Diego occurred among men aged between 21 and 52 years old ${ }^{12}$. This young key population accounted for $46.3 \%$ of all MSM in San Diego ${ }^{24}$. If PrEP was targeted to $30 \%$ of MSM between the ages of 21 and 52 years, it would avert 433 infections over 5 years (Fig. 2, see*), costing around $\$ 270$ million (Fig. S5, see*, and Table S3 in the Supplement, shaded box). By comparison,

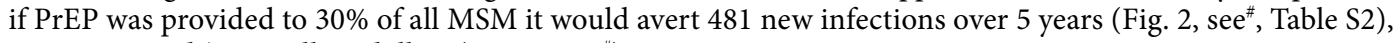
costing around $\$ 580$ million dollars (Fig. S5, see ${ }^{\#}$ ).

Since, a variety of combinations of TasP and PrEP coverage rates can reduce HIV incidence among MSM in San Diego, we performed a sensitivity analysis for age targeted versus non-targeted PrEP (Fig. 3). When TasP coverage rate was at $30 \%$ of all HIV-infected individuals and PrEP coverage rate at $30 \%$ of all susceptible MSM (i.e. non-targeted PrEP), we estimated that this would decrease the number of new infections from 638 to 355 after one year (Fig. 3A). This is similar to when TasP coverage rate remains at $30 \%$ and PrEP covers $30 \%$ of MSM between the ages of 21 and 52 years (i.e. age targeted PrEP), with number of new infections decreasing from 638 to 364 (Fig. 3B). However, the gross cost per infection averted was less for age targeted PrEP than non-targeted $\operatorname{PrEP}(\$ 558,000$ vs. $\$ 767,000$, Fig. 4).

Sexual Risk Compensation. To evaluate the potential impact of sexual risk compensation, we performed a two-way sensitivity analysis based on: (i) PrEP coverage rate, (ii) condom use, and (iii) average number of sexual 


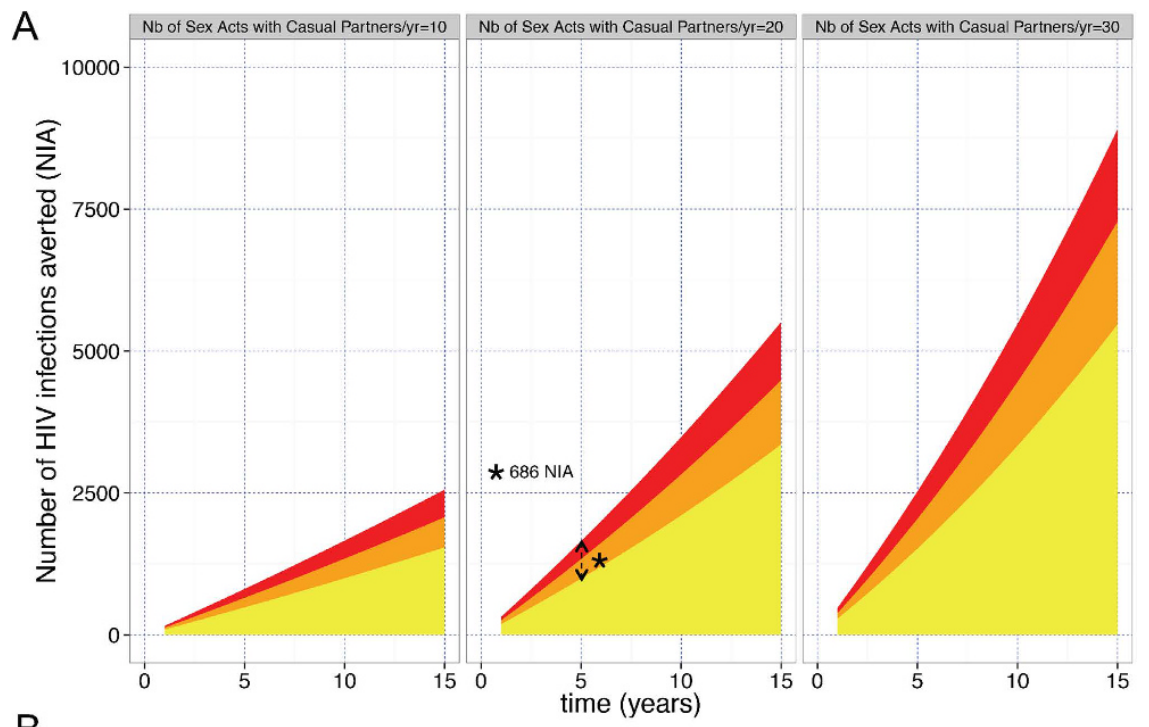

B

$\square 30 \%$ TasP coverage $\square 40 \%$ Tas $\mathrm{P}$ coverage $\square 50 \%$ Tas $\mathrm{P}$ coverage

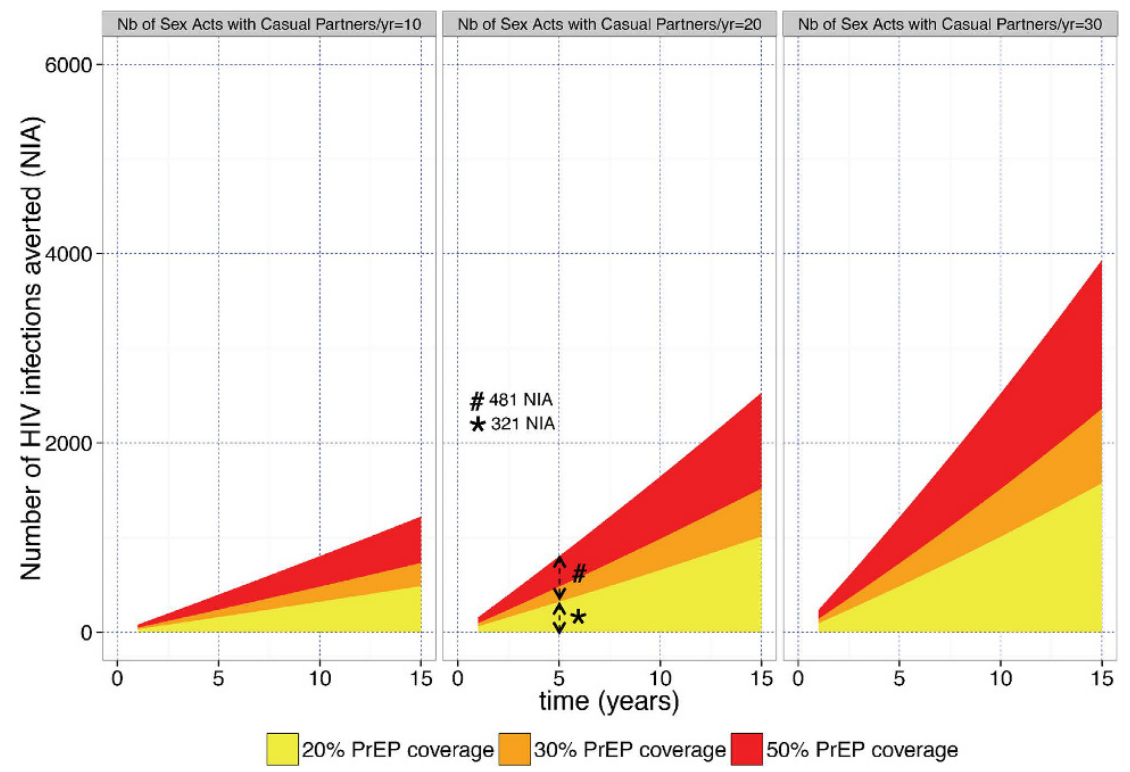

Figure 1. TasP coverage rate $(\mathbf{A})$ and PrEP coverage rate $(\mathbf{B})$ and estimated number of new HIV infections averted (NIA) among MSM in San Diego. The number of HIV infections averted (NIA) is proposed for: (A). Three different levels of TasP coverage rate are presented in yellow (30\%), orange (40\%) and red (50\%).

(B) Three different levels of PrEP coverage rate are presented in yellow (30\%), orange (40\%) and red (50\%). These analyses were based on an initial population size of 56,000 MSM, an HIV prevalence of $20 \%$ among MSM, a mean number of sex acts of 10, 20 and 30/year, and 60\% condom use, and are stratified by three levels of number of sex acts with casual partner (10,20 and 30/year).

acts with casual partner per year. First, if overall condom use decreased from $60 \%$ to $40 \%$ but PrEP coverage rate to all MSM was increased from $0 \%$ to $30 \%$, then in one year, the number of new HIV infections would increase from 499 to 591 (versus 394 if condom use had stayed at 60\%) (Fig. 4A). Similar to condom use, in one year the number of new HIV infections increased from 249 to 295 when sex acts with casual partners per year increased from 10 to 15 despite a PrEP coverage rate increase from $0 \%$ of $30 \%$ (Fig. 4B).

Combined TasP and PrEP. We then evaluated logical benchmarks to better understand what targets of PrEP and TasP coverage rates would be needed to make meaningful impacts on the San Diego epidemic. To reduce the number of HIV infections in San Diego by over half in only one year (638 vs. 317 new infections), simple calculations suggested that it would take an age targeted PrEP coverage rate of $40 \%$ and TasP coverage rate of $34 \%$, costing around $\$ 183$ million. To reduce the number of new infections in San Diego by two-thirds in 20 years would require at least half of MSM aged between 21 and 52 years to be covered by PrEP (age-targeted PrEP coverage rate) and $60 \%$ of HIV-individuals receiving effective ART (TasP coverage rate), costing around \$4.6 


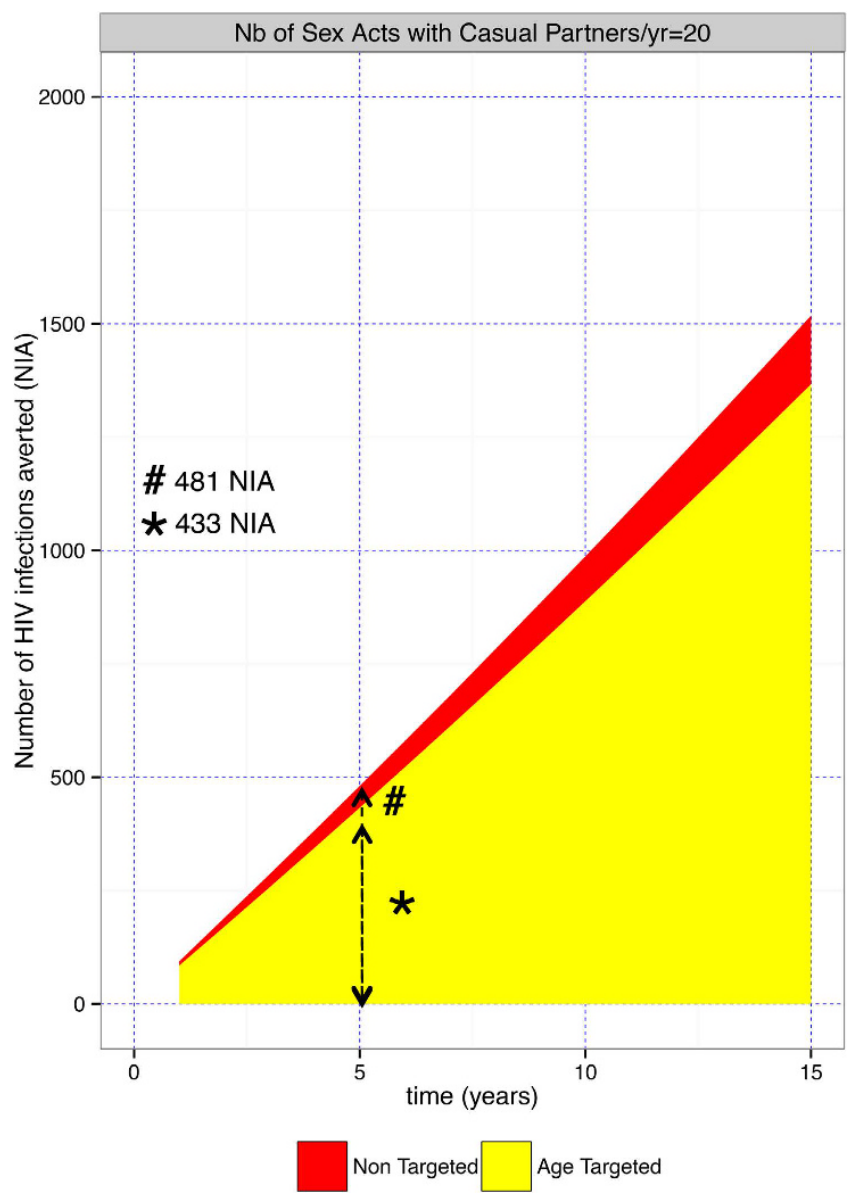

Figure 2. Number of new HIV infections averted (A) by targeting PrEP to MSM between the ages of 21 and 52 years. The number of new HIV infections averted are estimated for a PrEP coverage of $20 \%$ with an average number of sex acts with causal partner is 20 /year.

billion. In general, to prevent one HIV infection over one year, we estimated that $17 \mathrm{HIV}$-infected individuals should be treated with ART or 100 susceptible MSM should receive PrEP. All of these estimates depend on stable rates of sexual risk.

\section{Discussion}

This study developed an intuitive and flexible tool based on the main drivers of sexual HIV epidemics to provide targets of TasP and PrEP coverage rates needed to reduce HIV incidence. By modelling antiretroviral therapy benefits and gross direct costs, this model can also be used to maximizes the returns on investment in the HIV response as defined by the UNAIDS ${ }^{27}$. We evaluated this tool in the context of the well-characterized HIV epidemic in San Diego, which is predominantly driven by risk among MSM, similar to many metropolitan areas across the U.S. ${ }^{28}$. We have also provided this tool in a form that can be adapted for other communities.

In general, all HIV-infected individuals should receive ART as soon as possible for their own health, but this is clearly not occurring in the U.S. and in San Diego, where only about $25 \%$ and $30 \%$ of HIV-infected individuals are receiving effective $\mathrm{ART}^{6}$, Increasing these rates would also decrease 'community viral load'29 and thus overall transmission, i.e. TasP ${ }^{2}$. Similarly, PrEP use among young key populations is also low $^{7}$. Using the developed tool, we estimated what targets of TasP and PrEP coverage rates would be needed to impact HIV incidence. As an example, this tool estimated that it would require $60 \%$ TasP coverage rate and $50 \%$ PrEP coverage rate of MSM between the ages of 21 and 52 years to reduce the number of new infections in San Diego by $75 \%$ in 20 years. However, sexual risk compensation can jeopardize the benefits of prevention initiatives ${ }^{30}$, and we found the potential reduction in HIV incidence by PrEP and TasP coverage rates can be theoretically offset by increased risk behavior. Further, the provided assessment of combined TaSP and PrEP estimated the efficacy of PrEP as $70 \%$ among those individuals who adhere to prescribed to PrEP. We feel that this level of efficacy is conservative, given that the iPrEx trial demonstrated a much higher efficacy of prevention among those who regularly took the medication. However, this estimate may also be overly optimistic given that in the blinded randomized control iPrEx trial, a considerable number of participants did not adhere to their medication and thus the overall additional protection of PrEP was only 44\%. To this end, we have allowed the provided tool to be adjusted based on local estimates of PrEP efficacy. Similar to estimates on effectiveness to PrEP, our presented estimates for the 
Non-Targeted PrEP Coverage (NNI)

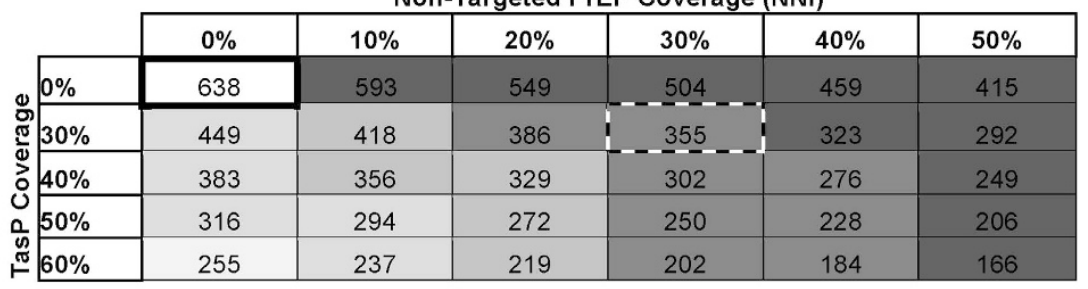

B. Age- Targeted PrEP (1 year)

\begin{tabular}{|c|c|c|c|c|c|c|c|}
\hline & \multicolumn{6}{|c|}{ Age Targeted PrEP Coverage (NNI) } \\
\hline & & $0 \%$ & $10 \%$ & $20 \%$ & $30 \%$ & $40 \%$ & $50 \%$ \\
\hline \multirow{2}{*}{$\begin{array}{l}\frac{\varpi}{\varpi} \\
\frac{5}{0} \\
\searrow\end{array}$} & $0 \%$ & 638 & 598 & 558 & 517 & 477 & 437 \\
\hline & $30 \%$ & 449 & 421 & 393 & 364 & 336 & 308 \\
\hline \multirow{3}{*}{$\begin{array}{l}0 \\
0 \\
0 \\
n \\
\sigma \\
\end{array}$} & $40 \%$ & 383 & 359 & 335 & 310 & 286 & 262 \\
\hline & $50 \%$ & 316 & 296 & 277 & 257 & 237 & 217 \\
\hline & $60 \%$ & 255 & 239 & 223 & 207 & 191 & 175 \\
\hline
\end{tabular}

Legend:

Cost per infection prevented

\begin{tabular}{|c|}
\hline$\$ 400-500,000$ \\
\hline$\$ 500-600,000$ \\
\hline$\$ 600-700,000$ \\
\hline$\$ 700-800,000$ \\
\hline$\$ 800-900,000$ \\
\hline
\end{tabular}

Figure 3. Number of new infections (NNI) and cost (shading) of Non-targeted (A) and Age Targeted (B) PrEP and TasP coverage rate in San Diego. The NNI is showed after 1 and 5 years for each scenario. Boxes are colored according to the cost in million USD per infection averted compared to a scenario without PrEP and TasP (white bold boxes).

effectiveness of condom use are overly optimistic and were presented as $100 \%$ effective, and perhaps more realistic at $80 \%$ effective, as condom use errors may be relatively frequent. Therefore, similar to PrEP effectiveness, we have allowed parameter to be adjusted in the tool.

The provided tool also offers a customizable simple costs calculator that can provide gross estimates of the cost of PrEP and TasP initiatives. It has been previously estimated that a prevention initiative would be cost saving if it cost less than $\$ 326,500$ per infection averted ${ }^{31}$. In the short term, most of our scenarios for TasP and PrEP had costs that were higher than this, but over time these costs, especially for TasP, decreased as more infections were averted (Fig. S4). As might be expected, we did find that targeting PrEP to MSM between the ages of 21 and 52 years could reduce the cost per new infection averted by $27 \%$ for a scenario with $30 \%$ TasP and $30 \% \operatorname{PrEP}$ coverage rates, although always remaining above $\$ 326,500$ per infection averted. We acknowledge that targeting PrEP to other groups, like very young MSM and minorities, may be even more effective ${ }^{12,32}$. These cost estimates are not exact, but we believe that this, perhaps overly simplified, tool can still provide meaningful costs estimates that can at least start the conversation around planning prevention initiatives. The strategic use of antiretroviral medications is important in an optimized effective investment approach in the HIV response ${ }^{27}$. Here, we provide a simple tool for practical planning of Public Health strategies allocating resources towards combinations of TasP and PrEP interventions. This tool is designed to provide an effective investment approach to maximize the returns on investment in the HIV response and enhance impact by tailoring efforts on key locations and populations, as appropriate.

The main limitation of this study is that the model is wrong. As Drs. Box and Draper stated in their book ${ }^{33}$, "Essentially, all models are wrong, but some are useful." While perhaps crude, our model used a flexible and simple structure to facilitate translation to other communities for accessible targets for TasP and PrEP coverage rates. An important part of HIV epidemics that this model ignores is the fact that the structure that underlies the transmission network is important ${ }^{34}$. As such, targeted prevention efforts, based on the structure of an epidemic, will likely provide the biggest impact ${ }^{35}$. Until such network information can be made available in real time for each community, we feel that this tool can provide good targets for TasP and PrEP coverage rate in local populations to reduce incidence. This tool also does not account for any migration of HIV-infected or susceptible individuals. Depending on the rates and directions of such population movement, this could have a negligible or large effect on HIV incidence. In San Diego, this might be especially pertinent given the 
A. PrEP coverage and condom use

PrEP Coverage

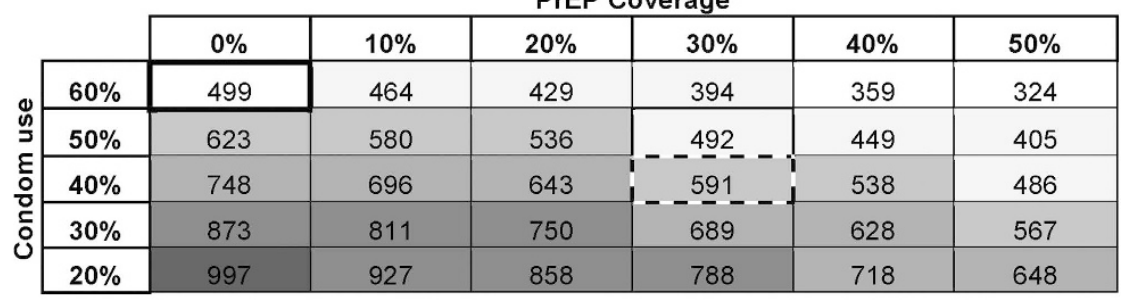

B. PrEP coverage and number of yearly sex acts with casual partners

PrEP Coverage

\begin{tabular}{|c|c|c|c|c|c|c|c|}
\hline & \\
\hline & & $0 \%$ & $10 \%$ & $20 \%$ & $30 \%$ & $40 \%$ & $50 \%$ \\
\hline \multirow{2}{*}{ 点 } & 10 & 249 & 232 & 214 & 197 & 179 & 162 \\
\hline & 15 & 374 & 348 & 322 & 295 & 269 & 243 \\
\hline \multirow{2}{*}{ 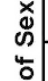 } & 20 & 499 & 464 & 429 & 394 & 359 & 324 \\
\hline & 25 & 623 & 580 & 536 & 492 & 449 & 405 \\
\hline 衣 & 30 & 748 & 696 & 643 & 591 & 538 & 486 \\
\hline
\end{tabular}

Legend:

HIV incidence compared to baseline

Decrease
Incidence $\left\{\begin{array}{c}0.5 \text { to } \leq 0.75 \text { fold } \\ 0.75 \text { to } \leq 1 \text { fold } \\ \text { Increase } \\ \text { Incidence }\end{array}\left\{\begin{array}{c}1 \text { to } \leq 1.25 \text { fold } \\ 1.25 \text { to } \leq 1.5 \text { fold } \\ 1.5 \text { to } \leq 2 \text { fold } \\ >2 \text { fold }\end{array}\right]\right.$

Figure 4. Number of new HIV infections (NNI) in relation to PrEP coverage rate and condom use (A) and average number of sex acts with casual partner $(\mathbf{B})$. In both scenarios, the number of new HIV infections are provided after one year considering $30 \%$ TasP coverage rate. Boxes are colored in grey scale in relation to the baseline NNI when PrEP coverage rate is $0 \%$ (white bold box), and darker colors represent change in NNI over baseline. Sexual risk compensation overcame the benefits of PrEP when the NNI is expected to be higher than the baseline NNI when PrEP was 0\%.

large bidirectional movement of people and HIV infections between San Diego, USA and Tijuana, Mexico ${ }^{35}$. Additionally, the model presented here does not allow for the prevalence of concurrent partnerships (i.e. partnerships that overlap in time $)^{36}$, which may a differential impact on epidemic growth patterns among key populations ${ }^{37}$. Another limitation of the model is that it does not account for changes in the lifespan of an infected individual who does or does not receive ART. In general, the more ART uptake, the longer is the lifespan of infected individuals. In fact, a person living with HIV who has access to and adheres to ART can expect a relatively normal lifespan ${ }^{38,39}$. Such changes in lifespan in those on ART could have a large impact on total costs. In addition, we expect that costs of TasP and PrEP fluctuate over time (e.g. manufacturer costs, third party payor negotiations, inflation, generic pricing). Therefore, we provide only gross cost estimates per year of the TasP and PrEP interventions (i.e. price per set interval) and have allowed these parameters to adjusted within the model by the user. More complex cost modeling would be needed to infer the total costs for changes in the lifespan by providing ART to infected individuals and preventing infections among susceptible individuals. Finally, more detailed and more accurate cost analyses of PrEP and TasP coverage rates, sexual risk compensation and effective measure/mathematical measure of concurrency ${ }^{40}$ will be needed in order to determine cost-effectiveness of the scenarios presented here.

In summary, we currently have the tools needed to eliminate local HIV epidemics with TasP and PrEP. We do not, however, have benchmarks of coverage rates needed for this endeavor. With a limited number of parameters and epidemiologic estimates, we provide here an easy-to-use and flexible tool to estimate the impact of TasP and PrEP coverage rates. Such a tool should assist public health officials and policy makers in their decisions about how to focus resources.

This tool is available online: http://gtzero.ucsd.edu/. 


\section{References}

1. Check Hayden, E. How to beat HIV. Nature 523, 146-148, doi: 10.1038/523146a (2015).

2. Cohen, M. S. et al. Prevention of HIV-1 infection with early antiretroviral therapy. N. Engl. J. Med 365, 493-505, doi: 10.1056/ NEJMoa1105243 (2011).

3. Grant, R. M. et al. Preexposure Chemoprophylaxis for HIV Prevention in Men Who Have Sex with Men. N. Engl. J. Med 363, 2587-2599, doi: 10.1056/NEJMoa1011205 (2010).

4. Gardner, E. M. \& Young, B. The HIV care cascade through time. Lancet Infect Dis 14, 5-6, doi: 10.1016/s1473-3099(13)70272-x (2014).

5. Skarbinski, J. et al. Human immunodeficiency virus transmission at each step of the care continuum in the United States. JAMA Intern Med 175, 588-596, doi: 10.1001/jamainternmed.2014.8180 (2015).

6. Epidemiology \& Immunization ServicesHIV/AIDS Epidemiology Unit of San Diego (HAEU). HIV/AIDS Epidemiology Report (2015) http://www.sandiegocounty.gov/hhsa/programs/phs/hiv_aids_epidemiology_unit/reports_and_statistics.html. (Date of access:02/25/2016).

7. Saberi, P. et al. Ambiguity, ambivalence, and apprehensions of taking HIV-1 pre-exposure prophylaxis among male couples in San Francisco: a mixed methods study. PLoS ONE 7, e50061, doi: 10.1371/journal.pone.0050061 (2012).

8. Granich, R. M., Gilks, C. F., Dye, C., De Cock, K. M. \& Williams, B. G. Universal voluntary HIV testing with immediate antiretroviral therapy as a strategy for elimination of HIV transmission: a mathematical model. Lancet 373, 48-57, doi: 10.1016/s01406736(08)61697-9 (2009).

9. Rosenberg, E. S., Millett, G. A., Sullivan, P. S., Del Rio, C. \& Curran, J. W. Understanding the HIV disparities between black and white men who have sex with men in the USA using the HIV care continuum: a modeling study. The lancet. HIV 1, e112-e118, doi: 10.1016/s2352-3018(14)00011-3 (2014).

10. Granich, R. et al. Expanding ART for treatment and prevention of HIV in South Africa: estimated cost and cost-effectiveness 20112050. PLoS ONE 7, e30216, doi: 10.1371/journal.pone.0030216 (2012).

11. Kahn, J. G., Marseille, E. A., Bennett, R., Williams, B. G. \& Granich, R. Cost-effectiveness of antiretroviral therapy for prevention. Curr. HIV Res 9, 405-415 (2011).

12. Morris, S. R. et al. Evaluation of an HIV nucleic acid testing program with automated Internet and voicemail systems to deliver results. Ann Intern Med 152, 778-785, doi: 10.1059/0003-4819-152-12-201006150-00005 (2010).

13. Bradley, H. et al. Vital Signs: HIV Diagnosis, Care, and Treatment Among Persons Living with HIV - United States. Center for Disease Control and Prevention (CDC) 63,1113-1117 (2014).

14. Patel, P. et al. Estimating per-act HIV transmission risk: a systematic review. AIDS (London, England) 28, 1509-1519, doi: 10.1097/ QAD.0000000000000298 (2014).

15. Røttingen, J.-A. \& Garnett, G. P. The epidemiological and control implications of HIV transmission probabilities within partnerships. Sexually transmitted diseases 29, 818-827 (2002).

16. Gebo, K. A. et al. Contemporary costs of HIV healthcare in the HAART era. AIDS (London, England) 24, 2705-2715, doi: 10.1097/ QAD.0b013e32833f3c14 (2010).

17. Juusola, J. L., Brandeau, M. L., Owens, D. K. \& Bendavid, E. The cost-effectiveness of preexposure prophylaxis for HIV prevention in the United States in men who have sex with men. Ann Intern Med 156, 541-550, doi: 10.7326/0003-4819-156-8-201204170-00001 (2012).

18. Macchione, N., W. W. J., Waters-Montijo, K. \& Ginsberg, M. (ed Health and Human Services Agency) (San Diego County, 2012).

19. Centers for Disease, C. \& Prevention. HIV prevalence, unrecognized infection, and HIV testing among men who have sex with men-five U.S. cities, June 2004-April 2005. MMWR Morb. Mortal. Wkly. Rep. 54, 597-601 (2005).

20. Bunnell, R. et al. Changes in sexual behavior and risk of HIV transmission after antiretroviral therapy and prevention interventions in rural Uganda. AIDS (London, England) 20, 85-92 (2006).

21. Smith, K., Powers, K. A., Kashuba, A. D. M. \& Cohen, M. S. HIV-1 treatment as prevention: the good, the bad, and the challenges. Curr Opin HIV AIDS 6, 315-325, doi: 10.1097/COH.0b013e32834788e7 (2011).

22. Jin, F. et al. "Any Condomless Anal Intercourse" is No Longer an Accurate Measure of HIV Sexual risk Behavior in Gay and Other Men Who have Sex with Men. Front Immunol 6, doi: 10.3389/fimmu.2015.00086 (2015).

23. Marrazzo, J. M. et al. HIV prevention in clinical care settings: 2014 recommendations of the International Antiviral Society-USA Panel. JAMA 312, 390-409, doi: 10.1001/jama.2014.7999 (2014).

24. U.S. Census Bureau - QuickFacts San Diego County, California (2015) https://www.census.gov/quickfacts/table/PST045215/06073. (Date of access:02/25/2016).

25. Flash, C. et al. Two years of Truvada for pre-exposure prophylaxis utilization in the US. Journal of the International AIDS Society 17, doi: 10.7448/ias.17.4.19730 (2014).

26. Menza, T. W., Hughes, J. P., Celum, C. L. \& Golden, M. R. Prediction of HIV acquisition among men who have sex with men. Sexually transmitted diseases 36, 547-555, doi: 10.1097/OLQ.0b013e3181a9cc41 (2009).

27. United Nations Programme on HIV/AIDS (UNAIDS)- Terminology Guidelines (2015) http://www.unaids.org/sites/default/files/ media_asset/2015_terminology_guidelines_en.pdf. (Date of access:05/15/2016).

28. Center for Disease Control and Prevention (CDC). HIV Risk, Prevention, and Testing Behaviors National HIV Behavioral Surveillance System, Men Who Have Sex with Men, 20 U.S. Cities, 2011. HIV Surveillance Report, Special Report 8 (2014). (Date of access:02/25/2016).

29. World Health Organization (WHO). Towards universal access: scaling up priority HIV/AIDS interventions in the health sector. Progress report 2011. Geneva, (2010). Available at: http://whqlibdoc.who.int/publications/2010/9789241500395_eng.pdf(Date of access:02/12/2016).

30. Blumenthal, J. \& Haubrich, R. H. Will risk compensation accompany pre-exposure prophylaxis for HIV? Virtual Mentor 16, 909-915, doi: 10.1001/virtualmentor.2014.16.11.stas1-1411 (2014).

31. Schackman, B. R. et al. The lifetime medical cost savings from preventing HIV in the United States. Med Care 53, 293-301, doi: 10.1097/MLR.0000000000000308 (2015).

32. Le, T. et al. Enhanced CD4+ T-cell recovery with earlier HIV-1 antiretroviral therapy. N. Engl. J. Med 368, 218-230, doi: 10.1056/ NEJMoa1110187 (2013).

33. Box, G. E. P. \& Draper, N. R. Empirical Model-Building and Response Surfaces (Wiley Series in Probability and Statistics) (1987).

34. Lewis, F., Hughes, G. J., Rambaut, A., Pozniak, A. \& Leigh Brown, A. J. Episodic sexual transmission of HIV revealed by molecular phylodynamics. PLoS Med 5, doi: 10.1371/journal.pmed.0050050 (2008).

35. Wang, X. et al. Targeting Prevention based on Molecular Epidemiology among Deeply Sampled Sub-Networks of Men Have who Sex with Men. Clin Infect Dis, doi: 10.1093/cid/civ526 (2015).

36. Watts, C. H. \& May, R. M. The influence of concurrent partnerships on the dynamics of HIV/AIDS. Mathematical biosciences 108, 89-104 (1992).

37. Morris, M. \& Kretzschmar, M. Concurrent partnerships and the spread of HIV. Aids 11, 641-648 (1997).

38. Samji, H. et al. Closing the gap: increases in life expectancy among treated HIV-positive individuals in the United States and Canada. PLOS ONE 8, e81355, doi: 10.1371/journal.pone.0081355 (2013).

39. Marcus, J. et al. In The annual Conference on Retroviruses and Opportunistic Infections (CROI) (Boston, 2016).

40. Sawers, L. Measuring and modelling concurrency. J Int AIDS Soc 16, 17431, doi: 10.7448/ias.16.1.17431 (2013). 


\section{Acknowledgements}

This work is supported by funds and grants from the following: the California HIV/AIDS Research Program (CHRP) Grant F13SD321, the Department of Veterans Affairs and grants from the National Institutes of Health: NIH Grants including AI106039, AI43638, AI074621, AI108351, AI036214, AI093163, AI100665, DA034978, AI036214; the Bettencourt-Schueller Foundation; the Max Kade Foundation, New York (Max Kade Postdoctoral Research grant); and the James B. Pendleton Charitable Trust.

\section{Author Contributions}

All authors had full access to all of the data in the study and take responsibility for the integrity of the data and the accuracy of the data analysis. Study concept and design: A.C., D.M.S., M.H. and S.J.L. Acquisition, analysis, or interpretation of data: A.C., D.M.S., M.H. and S.R.M. Drafting of the manuscript: A.C., D.M.S. Critical revision of the manuscript for important intellectual content: M.H., S.R.M. and S.J.L. Statistical analysis: A.C. Obtained funding: D.M.S. and A.C.

\section{Additional Information}

Supplementary information accompanies this paper at http://www.nature.com/srep

Competing financial interests: The authors declare no competing financial interests.

How to cite this article: Chaillon, A. et al. A practical online tool to estimate antiretroviral coverage for HIV infected and susceptible populations needed to reduce local HIV epidemics. Sci. Rep. 6, 28707; doi: 10.1038/ srep28707 (2016).

(c) (i) This work is licensed under a Creative Commons Attribution 4.0 International License. The images or other third party material in this article are included in the article's Creative Commons license, unless indicated otherwise in the credit line; if the material is not included under the Creative Commons license, users will need to obtain permission from the license holder to reproduce the material. To view a copy of this license, visit http://creativecommons.org/licenses/by/4.0/ 